\title{
A survey of RNA viruses in mosquitoes from Mozambique reveals novel genetic lineages of flaviviruses and phenuiviruses, as well as frequent flavivirus-like viral DNA forms in Mansonia
}

\author{
Ana Paula Abílio ${ }^{1,2^{*}}$ (D), Manuel Silva ${ }^{3}$, Ayubo Kampango ${ }^{1}$, Inácio Narciso ${ }^{4}$, Eduardo Samo Gudo ${ }^{1}$, \\ Luís Carlos Bernardo das Neves ${ }^{5}$, Mohsin Sidat ${ }^{2}$, José Manuel Fafetine ${ }^{6}$, António Paulo Gouveia de Almeida ${ }^{4}$ and \\ Ricardo Parreira ${ }^{3}$
}

\begin{abstract}
Background: Mosquito-borne diseases involving arboviruses represent expanding threats to sub-Saharan Africa imposing as considerable burden to human and veterinary public health. In Mozambique over one hundred species of potential arbovirus mosquito vectors have been identified, although their precise role in maintaining such viruses in circulation in the country remains to be elucidated. The aim of this study was to screen for the presence of flaviviruses, alphaviruses and bunyaviruses in mosquitoes from different regions of Mozambique.

Results: Our survey analyzed 14,519 mosquitoes, and the results obtained revealed genetically distinct insectspecific flaviviruses, detected in multiple species of mosquitoes from different genera. In addition, smaller flaviviruslike NS5 sequences, frequently detected in Mansonia seemed to correspond to defective viral sequences, present as viral DNA forms. Furthermore, three lineages of putative members of the Phenuiviridae family were also detected, two of which apparently corresponding to novel viral genetic lineages.

Conclusion: This study reports for the first-time novel insect-specific flaviviruses and novel phenuiviruses, as well as frequent flavivirus-like viral DNA forms in several widely known vector species. This unique work represents recent investigation of virus screening conducted in mosquitoes from Mozambique and an important contribution to inform the establishment of a vector control program for arbovirus in the country and in the region.
\end{abstract}

Keywords: Flaviviruses, Bunyaviruses, Mosquitoes, Viral DNA forms, Phylogenetic analysis, Mozambique

\footnotetext{
* Correspondence: anabilio1408@gmail.com

1 Instituto Nacional de Saúde (INS)-Ministry of Health (MISAU), Vila de

Marracuene, Estrada Nacional №1, Parcela №3943, P.O. Box: 264, Maputo, Mozambique

${ }^{2}$ Faculty of Medicine, Eduardo Mondlane University (UEM), Maputo, Mozambique

Full list of author information is available at the end of the article
}

C C The Author(s). 2020 Open Access This article is licensed under a Creative Commons Attribution 4.0 International License, which permits use, sharing, adaptation, distribution and reproduction in any medium or format, as long as you give appropriate credit to the original author(s) and the source, provide a link to the Creative Commons licence, and indicate if changes were made. The images or other third party material in this article are included in the article's Creative Commons licence, unless indicated otherwise in a credit line to the material. If material is not included in the article's Creative Commons licence and your intended use is not permitted by statutory regulation or exceeds the permitted use, you will need to obtain permission directly from the copyright holder. To view a copy of this licence, visit http://creativecommons.org/licenses/by/4.0/. The Creative Commons Public Domain Dedication waiver (http://creativecommons.org/publicdomain/zero/1.0/) applies to the data made available in this article, unless otherwise stated in a credit line to the data. 


\section{Background}

Vector-borne diseases caused by arboviruses such as the Rift Valley fever, dengue, chikungunya, Zika, or West Nile viruses (RVFV, CHIKV, DENV, ZIKV and WNV, respectively), represent emerging and expanding threats in sub-Saharan Africa, and remain a major burden to global health, despite increasing funding allocated for their control and eradication [1]. Every year, more than one billion humans are infected, many of who die from vector-borne viral diseases, and more than half of the world's population may currently be at risk of infection, particularly in low-income countries $[2,3]$.

Our knowledge of the diversity of the viral world has significantly expanded over the last decade. During this period, a large number of studies have shown that viruses are the most abundant biological entities on the planet and display a remarkable degree of genetic diversity and genomic plasticity $[4,5]$, and have also allowed us to bridge apparent phylogenetic gaps in the virosphere. This is especially true when viral surveys focus on rarely sampled taxa or infrequently visited biotopes, and revealing novel or divergent viral groups [6-10].

Invertebrates are among the animals most frequently sampled in recent viral surveys, and their viromes seem to include a large number of genetically diverse viruses [9]. Mosquitoes (Diptera: Culicidae) are clearly the invertebrates most commonly studied due to their role as vectors of pathogenic viruses to humans and other animals [11]. However, the viromes of mosquitoes have been shown not to be limited to the latter, many of which (e.g. dengue, yellow fever or Zika viruses) have become household names in recent times. In fact, mosquitoes also host a profusion of viruses that only infect invertebrate cells and are, therefore, regarded as insect-restricted [12-14]. On the other hand, viral surveys are still frequently carried out in association with disease outbreaks, or when identifiable factors increase the probability for an arbovirus to (re)emerge and/or rapidly disperse [11]. Moreover, since there is limited knowledge on the genetic diversity, and ecology, of viruses in their natural enzootic maintenance cycles, little is also known regarding the adaptive constraints ruling the evolutionary steps that determine arbovirus emergence from their sylvatic niches [15].

Mozambique is located in a region suitable to arbovirus outbreaks, and in recent times the country was affected by two dengue virus outbreaks, which occurred in the northern regions [16, 17]. Increasing evidence also suggest that the country may be endemic to other debilitating and lifethreatening arboviral threats including RVFV [18-20], DENV $[2,16,21]$ and CHIKV $[22,23]$. Moreover, historical and global risk projection have suggested that the country may also be suitable for the establishment of ZIKV [24-26], a virus recently linked to cases of microcephaly as well as many other neurological abnormalities in newly born infants [27]. Despite increasing evidence indicating the circulation of public heath-relevant arboviruses in Mozambique, the burden of the diseases they cause remains unknown. In addition, more than a hundred potentials arbovirus vectors have been identified in Mozambique, and these include Aedes spp, Culex spp, Mansonia spp and Anopheles spp [28-31], of which their role in maintaining arboviruses in nature remain to be elucidated.

The focus of this study was the detection, and analysis, of selected taxa of RNA viruses in different geographic regions in Mozambique. These regions display rich mosquito and wildlife faunas, as well as bioecological features that allow mosquitoes, wildlife, domestic animals and humans to coexist in close proximity. The viruses targeted in this viral survey included alphaviruses, flaviviruses, and different bunyaviruses. While our initial interest as far as bunyaviruses were concerned involved the detection of RVFV, in a subset of samples the viral screening also included detection of phlebovirus-like and orthobunyavirus genomes. The results obtained did not reveal the circulation of recognizable pathogenic viruses in wild-caught mosquitoes, but uncovered divergent phenuiviruses, as well as different lineages of insect-specific flaviviruses.

\section{Results}

The results presented in this work were based on the analysis of a total of 14,519 mosquitoes, collected in 3 regions of Mozambique (Fig. 1) during 12 successive collection campaigns, carried out between November/2014 and December/2015. The majority $45.55 \%$ ( $n=6614$ / $14519)$ of the screened mosquitoes were classified as Culex spp., followed by Anopheles spp. 27.16\% ( $n=$ 2943/14519) and Mansonia spp 25.22\% ( $n=3662$ / 14519). Mosquitoes were grouped into 351 pools, ranging from 1 to a maximum of 128 specimens, with the average of (approximately) 41 mosquitoes each. These were subsequently processed by RT-PCR for the detection of specific viral agents (such as RVFV), or groups of viruses (such as alphaviruses and flaviviruses).

\section{Analysis of flavivirus sequences}

The genomes of flaviviruses were targeted using the primers previously described by Vázquez et al., (2012), which reveal an amplicon with the expected mass of $\approx 1$ kbp [that on the Culex flavivirus strain CxFV-Mex07 reference sequence (EU879060) would define a section of the viral genome from coordinates 9800 to 9901] in the cDNA extracts prepared from $45 / 351$ pools (12.8\%). These results indicated the presence of flavivirus genome in 9 different species of mosquitoes from possibly 4 genera (Anopheles, Culex Coquillettidia, and Mansonia). A sample $(n=20)$ of these amplicons was sequenced, and BLASTn/x similarity searches unambiguously confirmed they had a flavivirus origin. Similarly, the mosquito 


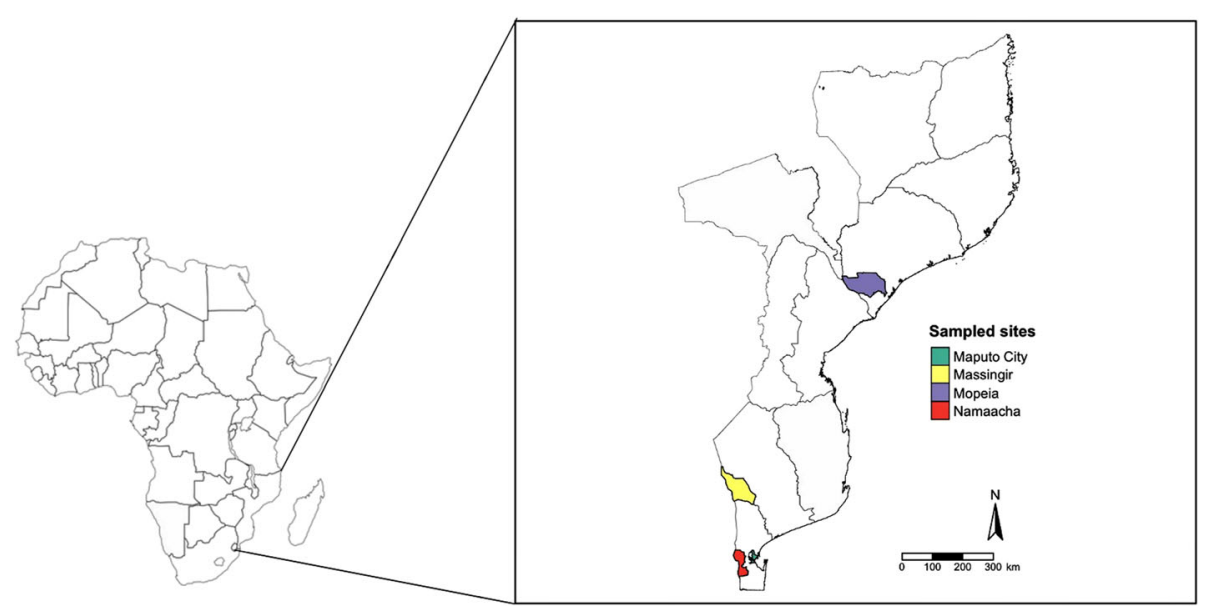

Fig. 1 Geographic coverage in Mozambique (west southern Africa) of the mosquito collections described in this report. The provinces indicated and the different municipalities where mosquito collections were carried out are color-coded

species of the pool of origin was confirmed by analysis of COI sequences in all but 5 pools, for lack of a PCR product. These corresponded to three of Ma. (Mnd) africana, and two of Ma. (Mnd) uniformis, all of which are very distinctive and clearly identifiable taxa. Table 1 lists all the viral sequences obtained in this study, as well as the date and location of collection of respective mosquito pools, their species, and respective accession numbers.

To further extend the characterization of the viral sequences obtained, a phylogenetic analysis was carried out using different methods. In all cases, the obtained phylogenetic trees indicated that none of the analyzed sequences had been amplified from bona fide arboviruses. Indeed, this is clearly revealed by their exclusion from the monophyletic cluster that assembles mosquito-borne and tick-borne flaviviruses in phylogenetic trees (cluster A in Fig. 2), the composition of which is shown in detail in the dotted box (indicated by the arrow). Conversely, all the sequences obtained in this study segregated within the large monophyletic group that assembles the so-called classical insect-specific flaviviruses, or cISF [12]. Furthermore, the analysis of the tree topologies obtained clearly suggested they did not group together in a single cluster, but rather segregated (i) either with other known viral sequences or (ii) formed independent genetic lineages. One of these lineages includes only sequences amplified from Anopheles spp. mosquitoes, while two others, also sharing a common ancestry, were mostly found in Mansonia spp. Unexpectedly, one of these sequences (LC462017) was obtained from a pool of mosquitoes identified as Culex ( $\mathrm{Cux}$ ) antennatus (pool Moz 182). However, the association of an apparently Culex-derived viral sequence with this group was considered debatable given its high similarity with the viral sequences amplified from Mansonia (see discussion). The above mentioned lineages of cISF include the Cuacua virus, previously identified in Mansonia sp. [29]. In this work, NS5-coding sequences $98-100 \%$ identical to those of the Cuacua virus were described both in Ma. africana and Ma. uniformis.

One of the other lineages of cISF identified is represented by a viral sequence obtained from $C q$. metallica which clustered with that of Nienokoue virus ( $\mathrm{NC}_{-}$ 024299) from Culex sp. However, these sequences share only $76.1 \%$ of sequence identity (as defined by Blast 2 sequence comparison), clearly below the $84 \%$ limit defined by Kuno and others [32] and, therefore, indicating that they represent distinct viral species. The remainder flavivirus lineages were detected in pools of Anopheles mosquitoes, four of which could be classified to the species level as $\mathrm{An}$. (Cel) pretoriensis and An. (Ano) coustani.

Curiously, the PCR amplification profiles of the flavivirus RT-PCR reactions frequently revealed (in agarose gels) the presence of an amplicon with approximately $0.5 \mathrm{kbp}$. This amplicon was observed in association with $31 / 351(8.8 \%)$ of the pools analyzed, by itself in $9 / 351$ (2.6\%) or in combination with the expected $1 \mathrm{kbp}$ DNA fragment in 22/351 (6.3\%). However, given its size, it would correspond to a deleted form of the NS5 coding gene suggesting (i) that it might have been amplified from defective viral genomes and/or (ii) rearranged forms of retro-transcribed viral DNA, possibly integrated in mosquito genomes as previously observed [33-35], and/or their resulting transcripts. The association of these smaller sequences with a flavivirus origin was clearly confirmed both by sequence homology searches (using BLASTn) and the reconstruction of phylogenies (Fig. 3a). All six $0.5 \mathrm{kbp}$ amplicons (indicated exclusively by NS5 $\Delta$ in Fig. 3a) that had been apparently obtained 


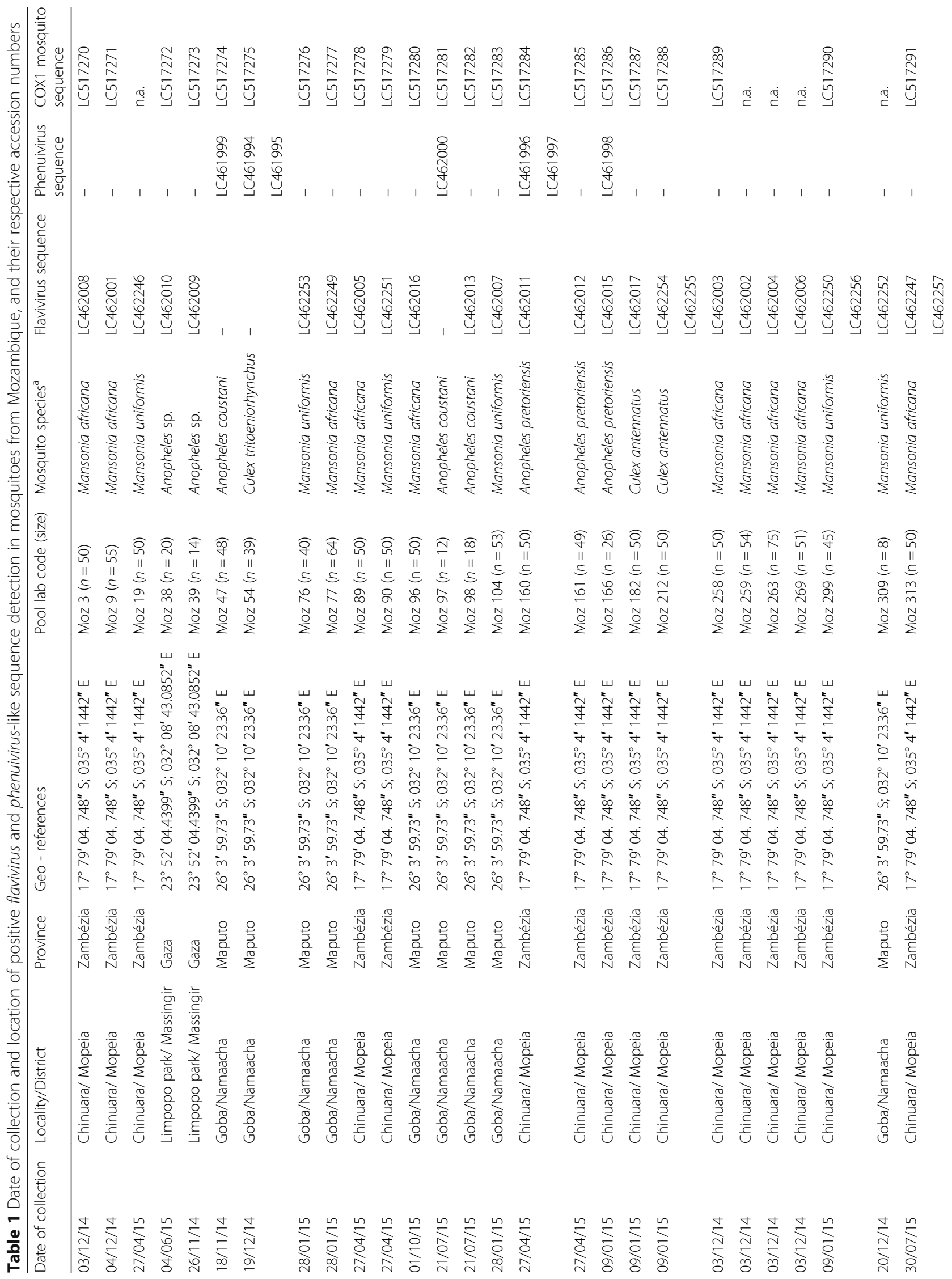




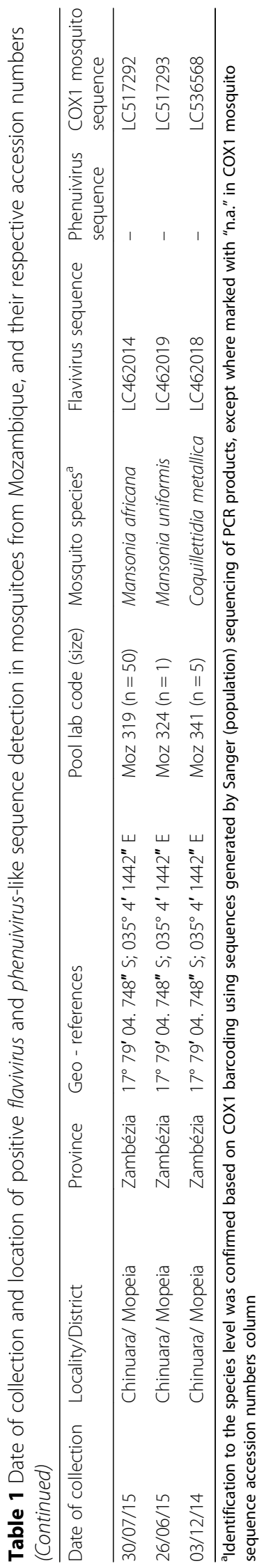




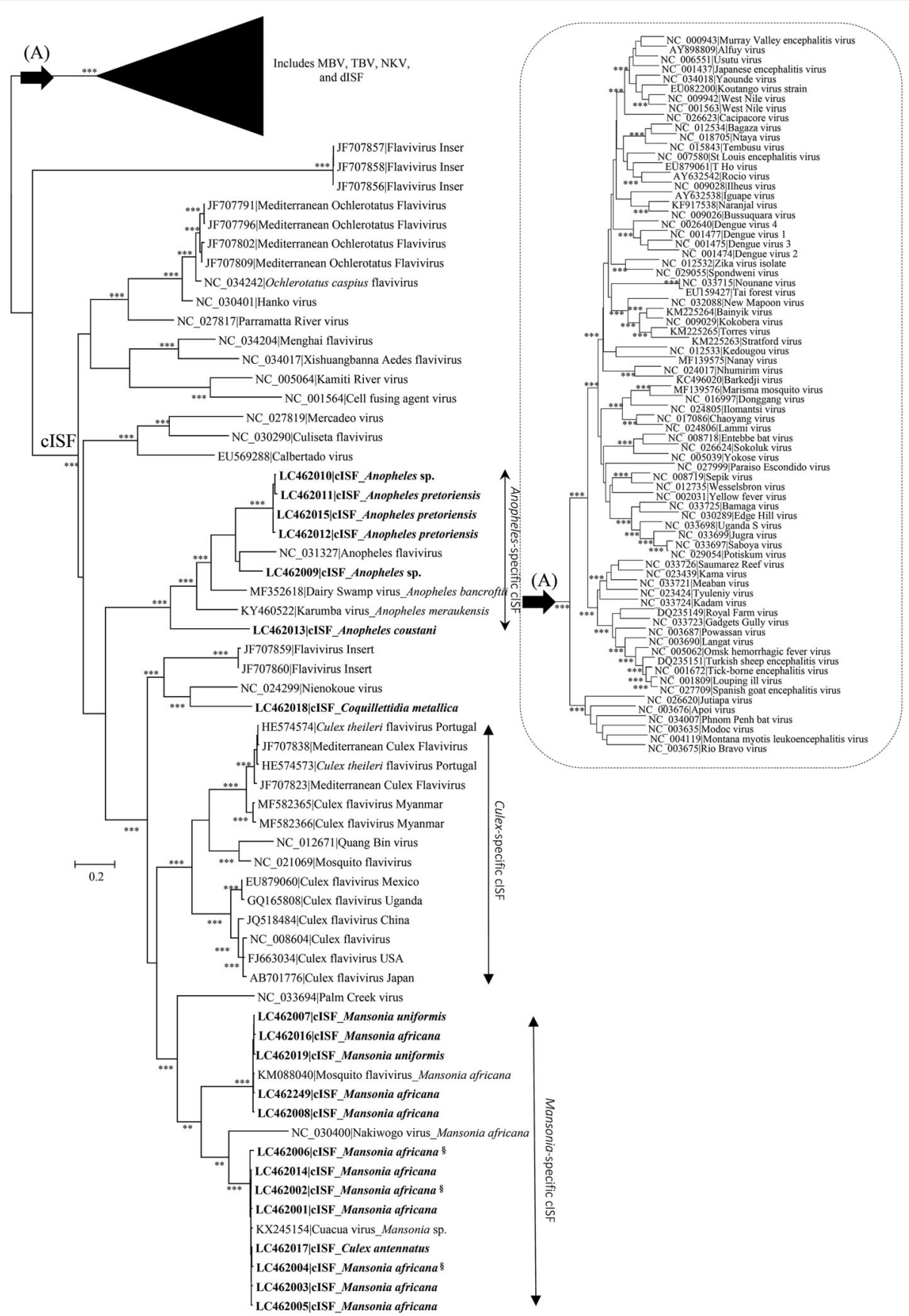

Fig. 2 a Phylogenetic analysis of flavivirus NS5 nucleotide sequences ( $\approx 1 \mathrm{kbp}$ per sequence). At specific branches, the number of "*" indicates the branch-support as revealed by the different phylogenetic reconstructions methods used, and assuming as relevant bootstrap values $\geq 75 \%$ (using 1000 resamplings of the sequence data in maximum likelihood analysis) and posterior probability values $\geq 0.80$ (when Bayesian approaches were used). One, two or three "*" would indicate that a given branch had been supported by one, two, or all the phylogenetic reconstruction approaches used in the amalysis (ML and Bayesian analysis using two sets of demographic priors). At the top of the tree, the collapsed monophyletic group including reference sequences from mosquito-borne viruses (MBV), tick-borne viruses (TBV), no known vector viruses (NKV), and dual-host associated insectspecific viruses (dISF), while the branches shown comprise the so-called classical insect-specific flaviviruses (cISF), is expanded at the right (b). The sequences described in this work are indicated in bold-face. All the sequences used are designated by their respective accession numbers|virus name. The size bar indicates the number of nucleotide substitutions per site. §-Mosquito species could not be confirmed by COI sequence 


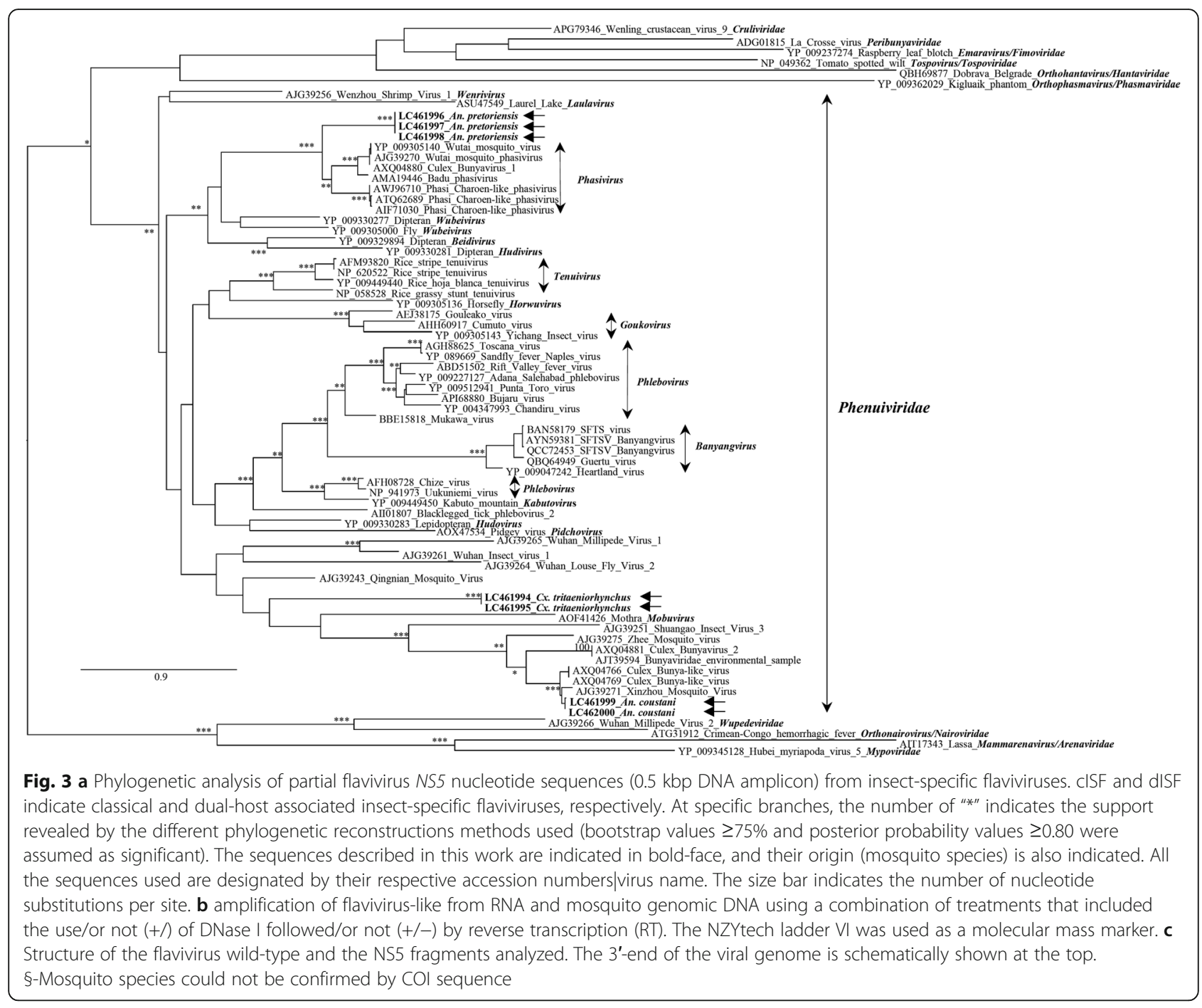

after amplification by RT-PCR from total RNA extracted from mosquito pools were not only clearly part of the cISF radiation but also clustered together in a single, and highly stable monophyletic cluster that subdivides into two subclusters (indicated by Mansonia-specific cISF/NS5 $\Delta$ in Fig. 3a). Moreover, these same $0.5 \mathrm{kbp}$ amplicons could also be obtained when total DNA was used as a template for PCR amplification, and no reverse-transcription had been performed, but when a DNase I treatment preceded reverse-transcription, no $0.5 \mathrm{kbp}$ amplification product was obtained (Fig. 3b). These results show that the origin of the frequently observed $0.5 \mathrm{kbp}$ fragment was not cDNA, but rather corresponded to viral DNA forms (vDNA) contaminating the RNA extracts. Three of these amplicons (indicated by the arrows in Fig. 3a), amplified from mosquito DNA pools of Ma. africana, Ma. uniformis and Cx. antennatus, were cloned and sequenced. Once again, the obtained sequences fell within the same monophyletic cluster. Moreover, when the structure of these DNA fragments was investigated, all of them revealed a similar architecture (Fig. 3c), combining both different sized deletions (down to $1 \mathrm{nt}$; indicated by $\Delta$ ) and point mutations.

\section{Screening of alphaviruses and bunyaviruses, and analysis of phenuivirus L-sequences}

Very different results were obtained when either Alphavirus-specific primers [36] or those targeting conserved sequences in the RVFV NSs coding-region [37] were used. In fact, neither of these sets of primers revealed the presence of the genomes of these viruses in any of the 351 pools of mosquitoes analyzed. Frequently, the use of the RVFV primers did result in the non-specific amplification of different sized PCR products, many of which were cloned and sequenced. In all cases (results not shown), the obtained sequences confirmed the nonviral origin of these amplicons. 
On the other hand, given the overwhelming diversity of the viruses that compose the recently proposed Order Bunyavirales, a decision was made not to restrict the screening of bunyaviruses to RVFV, but to extend it, in a smaller subset (43/351) of the pools of mosquitoes collected in different geographic areas of Mozambique, using Phlebovirus and Orthobunyavirus primers [38, 39]. This subset of 43 pools included the species Ae. (Adm) fowleri $(n=1)$, Ae. (Dic) adersi $(\mathrm{n}=1)$, Ae. (Muc) sudanensis $(n=1)$, Ae. (Neo) circunluteolus $(n=4)$, Ae. (Neo) mcintoshi $(n=2)$, Ae. (Ste) aegypti $(n=1)$, Ae. (Ste) metallicus ( $n=1)$, An.(Ano) coustani $(\mathrm{n}=2)$, An. (Ano) tenebrosus ( $\mathrm{n}=1)$, An. (Cel) funestus $(\mathrm{n}=1), A n$. (Ano) ziemani ( $\mathrm{n}=1), A n$. (Cel) pharoensis $(\mathrm{n}=1), A n$. (Cel) pretoriensis $(\mathrm{n}=2), C q$. (Coq) metallica $(\mathrm{n}=1), C x$. $(\mathrm{Cux})$ antennatus $(n=5), C x$. Cux ) tritaeniorhynchus $(\mathrm{n}=2)$, Cx. (Cux) neavei $(\mathrm{n}=2), C x$. (Cux) pipiens s.l. $(\mathrm{n}=2)$, Cx. (Cux) poicilipes $(\mathrm{n}=1), C x$. (Cux) zombaensis $(\mathrm{n}=1)$, Cx. sp. (1), Ma. (Mnd) africana $(n=3)$, and Ma. (Mnd) uniformis $(n=4)$, Mimomyia (Mim) mimomyiaformis $(\mathrm{n}=1)$, and one pool of $\mathrm{Ae}$. (Neo) sp.

Whereas the results that were obtained failed to reveal the presence of Orthobunyavirus genomes, in 5 pools, two of An. coustani (sequences LC461999, LC462000), two of An. pretoriensis (sequences LC461996, LC461997, and LC461998) and one of Cx. tritaeniorhynchus (sequences LC 461994 and LC46195) mosquitoes, a DNA fragment with the expected size was, indeed, amplified. All these amplicons were sequenced, but while BLASTn/x sequence searches did indicate a viral origin, unexpectedly they did not seem to have derived from bona fide Phlebovirus genomes, and this was confirmed by phylogenetic analysis using an assemblage of Phlebovirus, Bandavirus, Banyangvirus, and Goukovirus reference sequences. Regardless of the fact that the Phlebovirus group was paraphyletic, the sequences obtained from the analyzed mosquitoes from Mozambique did not cluster in any of the viral taxa in the tree, but rather formed 3 independent genetic lineages, as indicated by the arrows in Supplementary Fig. 1. The origin of these viral sequences was investigated using phylogenetic analysis of aligned amino acid sequences of the viral $\mathrm{L}$ protein from viruses classified within the different families in the Order Bunyavirales. The obtained results (Fig. 4) showed that, while all these sequences were clearly placed within the family Phenuiviridae, only two of them clustered with previously known viral references $[8,40,41]$, yet in a cluster with no assigned designation. The other five sequences, two amplified from a pool of $C x$. tritaeniorhynchus, and three others from pools of An. pretoriensis and An. coustani formed isolated genetic lineages, probably representing new unassigned genera.

\section{Isolation of viruses using $\mathrm{C} 6 / 36$ cells}

In an attempt to isolate some of the identified viruses, six filter-sterilized aliquots of macerates (Moz 39/Anopheles sp., Moz 54/Cx. tritaeniorhynchus, Moz 89/Ma. africana, Moz 97/An. coustani, Moz 98/An. coustani, and Moz 160/An. pretoriensis) were put in contact with subconfluent monolayers of $\mathrm{C} 6 / 36$ cells for virus isolation. Viral replication was allowed for 14 days (one blind passage was carried out at the end of the first week), after which the presence of viral genomes was verified by RTPCR using the same primers used for viral screening. At day three after the first blind-passage, three of these supernatants revealed evident CPE that included an apparent cell growth arrest accompanied by cell rounding, detachment from the flask surface, and sometimes clumping (Supplementary Fig. 2. Surprisingly, none of the primers used revealed the presence of any of the viruses detected during the viral screening. Moreover, as previously the isolation of Negev-like viruses had been associated, in our lab, to CPE similar to the one described above, RT-PCR with a series of primers designed for detection of several subsets of Nelorpiviruses (Carapeta et al., 2015), was performed. Once again, the presence of these viruses was not confirmed. Therefore, to the present day, the identity of the viruses isolated remains to be elucidated, which will be carried out using a metagenomic approach.

\section{Discussion}

In this report, a screening for different groups of RNA viruses targeting the detection of some of those previously shown (genome detection) or suggested (seroprevalence studies) to circulate in Mozambique [23, 29, $42,43]$. This analysis was carried out based on a oneyear sampling effort, that amounted to the screening (for viral genomes) of 14,519 mosquitoes from 3 regions of the country. As only female mosquitoes may serve as vectors of viruses to vertebrates, male mosquitoes were excluded from this viral screening. Although the detection of viral agents is facilitated when their presence is associated with visible clinical signs/symptoms in vertebrates, their screening in their natural hosts/vectors may have the advantage of signaling their circulation before any cases of clinical disease, or seroprevalence, are detected. Moreover, a viral screening effort based on the identification of foci of disease cases only discloses the circulation of pathogenic viruses, and these have been shown to represent only a part of the virome of mosquitoes [11-14].

The molecular screening that was carried out did not reveal the presence of RVFV or any recognizable pathogenic alphaviruses, bunyaviruses or flaviviruses. These include viruses such as DENV, ZIKV, CHIKV, o'nyong nyong, Sindbis or Middelburg [36]. While the absence of alphaviruses in this viral screening may be intriguing, we 


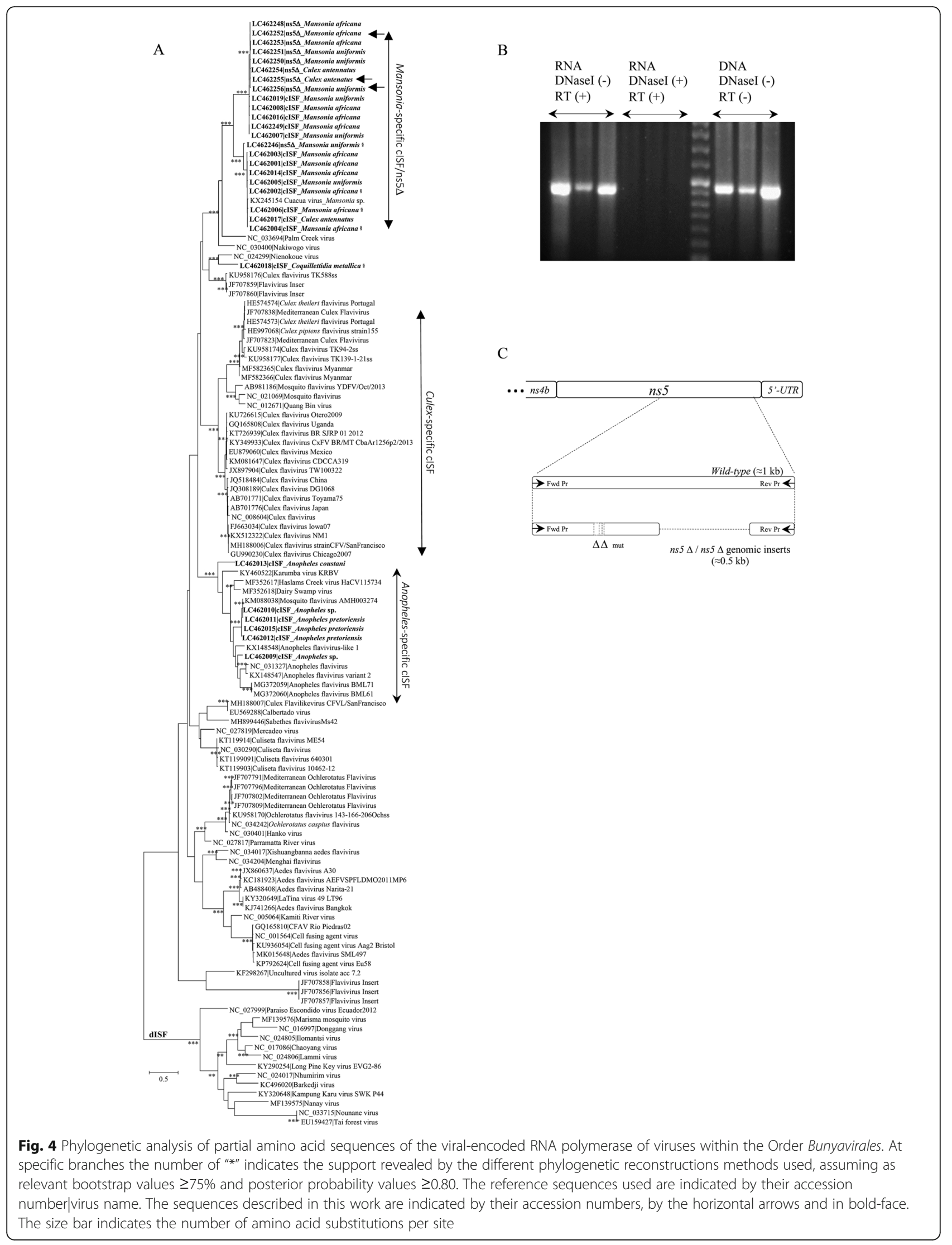


must bear in mind that unlike other virus groups (e.g. flaviviruses), alphavirus ISVs have, with exceptions [44, 45], been less frequently reported in viral surveys, while pathogenic alphaviruses such as CHIKV are usually associated with Aedes mosquitoes which were clearly underrepresented in our screening. Furthermore, no Orthobunyavirus sequences were ever detected in a small sample of the pools analyzed $(n=43$; the same subset of pools where a survey for Phlebovirus genomes was also carried out).

On the contrary, the use of a highly degenerate flavivirus-specific primer set [46] confirmed the presence of multiple genetic lineages of flaviviruses in a large number of pools of mosquitoes. Despite the fact that not all of the obtained amplicons were sequenced, those for which a sequence was obtained were found to segregate in the cISF radiation.

The different genetic lineages of viral NS5 sequences were apparently associated with multiple species from 4 genera, supporting the perception that cISF are widespread in the natural populations of mosquitoes. Some of these NS5 sequences seemed to segregate away from previously described viral assemblages and formed isolated branches in phylogenetic trees. Others were joined in clusters with multiple operational taxonomic units that were never exclusively associated with a single species of mosquitoes, adding to the possibility that cISF may not host species-restricted [47, 48]. However, while phylogenetic analysis did suggest a Culex origin for one of the sequences (LC462017), given the fact that it was almost identical to many others amplified from Mansonia mosquitoes, its association with Culex mosquitoes is disputable. Furthermore, although a molecular confirmation of the identity of these mosquitoes was obtained by $C O I$-sequence analysis, the sequencing strategy used (Sanger) is a population-approach that only reveals the sequence of the most abundant molecular form in a PCR-product, while minor variants fail to be detected. Therefore, we cannot formally exclude the possibility that sequence LC462017 may have been derived from one/a small number or even body-parts of non-Culex mosquitoes (possibly Mansonia) originally present in the pool in question.

Surprisingly, in a high number of pools of Mansonia spp. $(n=29)$ in one pool of Anopheles sp. and another of Culex sp. mosquitoes, the flavivirus-specific primers used generated a smaller than expected PCR product, with approximately half the size $(\approx 0.5 \mathrm{kbp})$. The analysis of some of these smaller amplicons showed that they corresponded to defective versions of the RdRp coding sequence and their origin was found to be DNA (vDNA), rather than RNA. For all those cases where a nucleotide sequence could be obtained, a shared ancestry between the latter and bona fide viral NS5 sequences (obtained by RT-PCR) was also revealed.
While we cannot ascertain, at this stage, whether the flavivirus vDNA forms are present as part of the host genome (endogenized), or whether they exist in the form of a stable extra-chromosomal DNA element, flaviviruslike sequences have been known to occur in the genome of mosquitoes for over a decade, especially in association with Aedes mosquitoes [33, 34]. While the sequence of a vDNA amplicon amplified from Anopheles could not be obtained due to technical difficulties, the fact that virtually identical vDNA sequences could be amplified from DNA extracts of Mansonia and Culex mosquitoes is hard to explain given the evolutionary divergence of these taxa. Moreover, while these vDNA forms could result from exposure of these mosquitoes to a common source of viruses, the possibility of a contamination of pools of Culex mosquitoes with even a limited amount of the highly abundant Mansonia specimens, cannot be discarded.

Whereas the presence of bacterial symbionts of mosquitoes can alter the competence of mosquitoes for transmission of pathogenic viruses [49], to what extent the same applies to the persistent presence of insectspecific viruses in insect cells is still open to discussion. However, the highly rearranged NS5 sequences found in this study seem to exclude the possibility that translation of an RNA transcribed from them might result in an active protein. In any case, they could participate in the establishment of persistence viral infections by controlling the siRNA response, as previously suggested [50].

Given the a priori specificity of the primers used for the screening of Phlebovirus sequences, the observation of a specific amplicon in association with 5 pools of 2 different species of Anopheles (An. coustani and An. pretoriensis) and one species of Culex (Cx. tritaeniorhynchus) mosquitoes suggested that these viruses might have been detected. However, the different phylogenetic analyses were congruent in showing (i) their inclusion in the Phenuiviridae family, (ii) but their exclusion from the Phlebovirus genus, (iii) and their separation into three genetic lineages. Two of these sequences did segregate in a stable monophyletic cluster defining a genetic lineage with no assigned designation, but that included sequences previously detected in other studies $[8,40$, $41]$, while the other five formed two genetic lineages with no associated references.

Although no recognizable pathogenic viruses were identified in the course of this work, this may result from a combination of multiple factors that include sampling bias. In fact, collections did not focus on settings where DENV/ZIKV/CHIKV were previously known to circulate in Mozambique [23, 42, 43], but rather on areas where RVFV had been detected [18, 19]. On the contrary, Mansonia and Culex mosquitoes clearly dominate the collections in the 3 provinces of Mozambique that 
were the focus of this study. However, pathogenic flavivirus such as the Spondweni virus (the closest known relative to ZIKV), have indeed been isolated from $M a$. africana and Ma. uniformis [11], as well as from Culex quinquefasciatus mosquitoes in Haiti [51]. Association of other pathogenic flaviviruses with Mansonia sp. mosquitoes include the S. Louis encephalitis and West-Nile viruses (which also use Culex sp. for their natural maintenance), alphaviruses (including Venezuelan equine encephalitis virus), orthobunyaviruses [52], and phleboviruses, including RVFV [11].

While sampling bias may partially explain the absence of some of the arboviruses that have been previously shown to circulate in Mozambique, other factors may also explain the results obtained. These include a low natural incidence of arboviruses in the areas where mosquitoes were collected, or the concurrent absence of recorded cases of human/animal disease cases associated to the circulation of viruses such as RVFV during the mosquito collection periods. Furthermore, a technical limitation of this study is associated with the use of a less technologically advanced virus detection approach based on convectional RT-PCR, as opposed to addressing viral screening with a bona fide metagenomic experimental design combined with the use of NGS sequencing methods. To the best of our knowledge, this study and the previous detection of ISF in Mansonia spp [29], are the only recent virus surveys using mosquitoes from Mozambique, and clearly demonstrates the dire need for such surveys that might clarify their epidemiology.

The attempted isolation of some of the viruses identified in this work in insect cells was not successful. This fact may probably result from a combination of factors that include the use of only one blind passage and a single cell-line. Indeed, while C6/36 cells have been extensively used for the isolation of ISVs they may not be susceptible and well as permissive to all insect viruses. In this regard, it should be added that some ISF seem to be restricted to theirs hosts [53], and this may indicate that use of C6/36 cells, although convenient, may not have been ideal. For more clarification further analysis involving cell culture attempts using a larger number of cell lines originating from different species of mosquitoes is recommended. While a very short blind-passage history may have compromised the production of a high titer viral suspension, in truth the exact same RT-PCR protocols were used to screen the presence of viral genomes in mosquito macerates and culture supernatants. Moreover, only after a single blind-passage, $50 \%$ of the cultures did evidence unambiguous CPE. Taking into account the protocol used, this CPE most probably was due to viral replication.

The nature of these viruses is currently under investigation. Furthermore, bioinformatics investigations for producing the unidentified CPE observed in inoculated cells is encouraged for better understanding the prevalence of insect-specific viruses in many genera of mosquitoes $[54,55]$. While these efforts should be ideally addressed using unbiased and high-throughput experimental approaches (metagenomics/NGS), the direct screening of other frequently found ISVs, including alphamesoniviruses [56] could also be pursued using a direct targeting strategy with taxon-specific primers.

\section{Conclusion}

This study reports for the first-time novel insect-specific flaviviruses and phenuiviruses, as well as frequent flavivirus-like viral DNA forms in several widely known vector species. While a large diversity of ISVs have been found on a global scale $[57,58]$ in association with a plethora of insect hosts, this work extends the results of the sole study that had, up to the present day, revealed their presence in Mozambique [29]. Although this survey did not disclose the circulation of pathogenic arboviruses, it confirmed the circulation of different RNA viruses that are present in mosquitoes from Mozambique. This article represents our professional endeavor to help to elucidate and provide higher resolution information on arboviruses vectors hotspot, transmission dynamics and routes in Mozambique and is of utmost importance to inform the establishment of a vector control program for arbovirus in the country and other region sharing the same pattern.

\section{Methods}

\section{Study area and mosquito collection}

A total of 14,519 mosquitoes were collected in rural settings in Mozambique (located in west southern Africa) between November 2014 and December 2015 as part of the work of Abílio, AP (In Preparation) at Massingir (in the province of Gaza), Namaacha (in the province of Maputo), and Mopeia (in the province of Zambézia) (Fig. 1). The general biotypes for Goba were savanna with medium grassland located around 10 to $500 \mathrm{~m}$ from a water stream. Collection sites in Massingir and Mopeia corresponded to forest environments located closed to the Lipompo and Zambezi rivers, respectively. The mosquitoes were collected using a combination of sampling methods that included indoor resting, tent collections and those carried out using $\mathrm{CO}_{2}$-baited miniature $\mathrm{CDC}$-light traps. These mosquitoes were stored in dry ice, and then transported to the laboratory for sorting and taxonomic identification using keys proposed by Gillies and Coetzee [59] and Jupp [60]. The manipulations of specimens for identification were carried out at temperatures close approximate to $0{ }^{\circ} \mathrm{C}$ under a stereomicroscope equipped with an ice block. Male and blood-fed specimens were 
excluded from this study. All samples were then stored at $-80^{\circ} \mathrm{C}$ until viral screening was carried out.

\section{Preparation of mosquito homogenates, and nucleic acid extraction}

The preparation of mosquito homogenates was based on a preliminary grouping of the collected and identified specimens in pools according to their species, sex, geographic origin, and blood-fed status. These mosquitoes were mechanically disrupted in $15 \mathrm{ml}$ Falcon tubes by vortexing using glass-beads and aluminum oxide in $1 \mathrm{ml}$ of phosphate buffer saline (PBS) buffer. After 3 pulses of $1 \mathrm{~min}$ (with $30 \mathrm{~s}$ breaks on ice), the mosquito macerates were clarified by centrifugation, as previously described (Carapeta et al., 2015). RNA, as well as DNA, were extracted from $200 \mu$ of clarified mosquito homogenate using $\mathrm{NZYol}^{\circ}$ (NZYTech, Portugal), as indicated by the supplier. The extracted RNA was dissolved in $30 \mu \mathrm{l}$ nuclease-free water, while the obtained DNA sediments were dissolved in 40-100 $\mu \mathrm{l}$ using a 1:1 mixture of $8 \mathrm{mM} \mathrm{NaOH}$ and TE buffer (Tris $100 \mathrm{mM}$, EDTA $1 \mathrm{mM}, \mathrm{pH}=7$ ).

\section{Viral genome detection}

The extracts of total RNA served as a template for the synthesis of cDNA, that was carried out with the NZY First-Strand cDNA Synthesis Kit (NZYTech, Portugal) using random hexamers, and a thermal profile including $10 \mathrm{~min}$ at $25^{\circ} \mathrm{C}, 45 \mathrm{~min}$ at $52^{\circ} \mathrm{C}$ and $10 \mathrm{~min}$ at $80^{\circ} \mathrm{C}$ (for enzyme inactivation), followed by treatment with $\mathrm{RNa}$ $\operatorname{seH}\left(20 \mathrm{~min}\right.$ at $\left.37^{\circ} \mathrm{C}\right)$.

Detection of flavivirus NS5 sequences (encoding the viral RNA-dependent RNA polymerase, or $\mathrm{RdRp}$ ) was carried out using previously described primers and reaction conditions [46]. A generic PCR method using degenerate primers targeting the $n s P 4$ gene (also encoding the viral $\mathrm{RdRp}$ ) was used to detect the presence of the genomes of alphaviruses [36], while RVFV genomic NSs coding sequences were tentatively detected as previously described [61]. Finally, the presence of phleboviruses and orthobunyaviruses $\mathrm{L}$ sequences (also encoding an RdRp) was investigated using the ppL1/ppL2 sets of primers/reaction conditions previously described by Matsuno and others [38] and the technical modifications suggested by Pereira and others [62], or as defined elsewhere [39]. Nelorpivirus detection was carried out as previously defined [63]. All the PCR primers and thermal profiles used are listed in Supplementary Table 1. PCR amplifications were carried out using NZYTaq 2X Green Master Mix (NZYTech, Portugal). The obtained amplicons were purified and directly sequenced or cloned in either pGEMT-easy ${ }^{\circ}$ (Promega, USA) or pNZY28-A using the NZY-A PCR cloning kit (NZYTech, Portugal), followed by DNA sequencing of individually purified plasmid recombinant-DNA molecules.

\section{Cell culture and virus isolation}

Aedes (Ste) albopictus C6/36 cell line was used for virus isolation. Cells were maintained at $28^{\circ} \mathrm{C}$ (in the absence of $\mathrm{CO}_{2}$ ) in L-15 Leibovitz Medium (Lonza, USA) supplemented with $10 \%$ heat-inactivated fetal bovine serum (FBS) (Lonza, USA), $2 \mathrm{mML}$-glutamine (Gibco BRL, USA), $100 \mathrm{U} / \mathrm{ml}$ penicillin and $100 \mu \mathrm{g} / \mathrm{ml}$ streptomycin (Gibco BRL, USA) and $1 \times$ tryptose phosphate broth (AppliChem $\mathrm{GmbH}$, Germany). Approximately $500 \mu \mathrm{l}$ of filter-sterilized mosquito homogenate were diluted in the same volume of phosphate buffered saline (PBS), and inoculated onto semi-confluent layers of C6/36 cells grown in T25 culture flasks (Nunc, Denmark). After $1 \mathrm{~h}$ at room temperature (for viral adsorption), the viral inoculum was removed, $5 \mathrm{ml}$ of L-15 Leibovitz Medium (2\% FBS) was added to each flask, and the cell cultures were incubated at $28^{\circ} \mathrm{C}$ for a week. Culture supernatants collected after a single blind-passage were used as viral stocks and stored at $-80^{\circ} \mathrm{C}$. Cytopathic effect (CPE) was determined by microscopic observation of the inoculated cell cultures.

\section{DNA sequencing and genetic analyses}

Multiple alignments of nucleotide (nt) or amino acid (aa) sequences were performed using the iterative GINS-I and E-INS-I methods as implemented in MAFFT vs. 7 [64] followed by editing using both GBlocks [65], and visual inspection. The multiple sequence alignments of nucleotide sequences were systematically verified to ensure the correct alignment of homologous codons using BioEdit 7.0.5 [66].

Phylogenetic trees were constructed using both Maximum Likelihood (ML) and Bayesian approaches. The best-fitting evolutionary models used were those suggested by JModeltest2 (Darriba et al., 2012) and W-IQtree (Trifinopoulos et al., 2016) for the analysis of $n t$ $($ GTR $+\Gamma+\mathrm{I}$ : GTR-General Time Reversal, $\Gamma$-Gamma distribution, I-proportion of invariant sites) or aa alignments (LG + Г: Le-Gascuel, $\Gamma$-Gamma distribution). Phylogenetic analyses based on the ML optimization criterion were carried out using the Mega 6.0 software [67], and the stability of the obtained tree topologies assessed by bootstrapping with different re-samplings of the original aligned positions (1000 for nt alignments, 100 for aa sequence data). Phylogenetic reconstructions following a Bayesian approach were carried out by running two independent Markov chain Monte-Carlo (MCMC) analyses using BEASTv1.7.5 [68], assuming a relaxed uncorrelated lognormal molecular clock model [69] as suggested by the ML Clock Test implemented in Mega 6.0. The MCMC chains were run until 100,000,000 states were sampled using both logistic population growth and Gaussian Markov random field/GMRF skygrid demographic priors. The Tracer software (http://beast.bio.ed.ac.uk/tracer) was used to diagnose stationarity and adequate $(>300)$ effective sample size 
(ESS). The trees were logged on every 5000th MCMC step, and the tree sample was summarized using TreeAnnotator v1.8.3 as maximum clade credibility (MCC) trees, with median heights used as the node heights in the tree, after discarding $10 \%$ of them as burn-in. The FigTree v1.4.2 software was used to visualize the phylogenetic trees (http://tree.bio.ed.ac.uk/software/figtree/).

The molecular confirmation of the morphological identifications of mosquitoes was carried out on the basis of the analysis of the barcoding section (from positions 58 to 705 encoding the $\mathrm{N}$-terminal section of the mitochondrial cytochrome oxidase subunit I - COI) essentially using Bold Systems-v4 (available at http://www.boldsystems.org/).

The nt sequences obtained in the course of this study were deposited in the GenBank/ENA/DDBJ databases under accession numbers LC461994-LC462019, and LC462246-LC462257, and LC517270-LC517293. The reference sequences used for analyses presented in this manuscript where directly downloaded from the public sequence databases. Whenever necessary, nt sequence similarity searches were carried out using BLASTn, and BLASTx (https://blast.ncbi.nlm.nih.gov/Blast.cgi).

\section{Supplementary information}

Supplementary information accompanies this paper at https://doi.org/10 1186/s12866-020-01905-5.

Additional file 1: Supplementary Figure 1. Microscopic observation of C6/36 cells mock-infected cells (A; 300x), or after infection (day 3) with viruses present in three independent pools of Ma. uniformis, An. ziemani, and An. pretoriensis mosquitoes collected in Mozambique.

Additional file 2.

Additional file 3: Supplementary Table 1. PCR primers and thermal profiles used in this work.

\section{Abbreviations}

DNA: Deoxyribonucleic acid; RNA: Ribonucleic acid; cDNA: complementary DNA; PCR: Polymerase chain reaction; RT-PCR: Reverse transcriptase-PCR

\footnotetext{
Acknowledgments

We also thank the staff from the Medical Entomology Laboratory from the National Institute of Health; Staff from the Biotechnology Center from Eduardo Mondlane University; Livestock Services from Zambézia Province; Livestock Services from Maputo Province; Staff from Limpopo National Park; Dr. Rogério Lopes Henriques owner of the Cuacua Lodge in Zambezia province and Dr. Telma Loforte owner of the Goba farm in Maputo Province including their staff; Health Services from Caia District; Staff from Instituto de Higiene e Medicina Tropical (IHTM)/Universidade Nova de Lisboa (NOVA) for their support during fieldwork, equipment and laboratory analysis for this work.
}

\section{Authors' contributions}

APA, JMF, LCBN, MS2 and APGA - conceived the investigations; APA, MS2, ESG, APGA and LCBN - Funding acquisition for the project; APA, RP and APGA - Methodology of the study; APA, JMF, LCBN and APGA - collected and performed pre-identified of the specimens; APA, AK and APGA - Confirmation of specie identifications; APA, MS1, IN, APGA, RP - Molecular and Phylogenetic Analysis; ESG, JMF, RP - Reagent and Materials. APA, APGA and RP - Data analysis. APGA and RP - Data curation and Validations. APA, APGA and RP - Writing original draft. APA, MS1, AK, ESG, MS2, LCBN, JMF, APGA and RP - Writing, revised and approved the final manuscript. The authors have read and approved the manuscript.

\section{Funding}

This study received main funding from Wellcome Trust [Grant WT087546MA] as part of main author SACIDS RVF PhD fellowship.

Additional funding was provided for travel and laboratory analyzes in country and abroad by BICMINS Project and NPHI-Phase-II from the National Institute of Health of Mozambique through a cooperative agreement number [5NU14GH001237-03-00]. Global Health and Tropical Medicine Center is funded through FCT contract UID/Multi/04413/2013.

The funding institutions had no role in study design, data collections, data analysis, manuscript writing and decision to publish.

\section{Availability of data and materials}

The datasets used and/or analyzed during the current study are available from corresponding author on reasonable request.

\section{Ethics approval and consent to participate}

The Ethics Committee from the Faculty of Medicine of Eduardo Mondlane University approved the study (Ref \#: CIBS FM\&HCM/15/2018). All head of the household or properties owners provided written informed consent prior to participation.

\section{Consent for publication}

Not applicable.

\section{Competing interests}

The authors declare that they have no competing interests.

\section{Author details}

${ }^{1}$ Instituto Nacional de Saúde (INS)-Ministry of Health (MISAU), Vila de Marracuene, Estrada Nacional Nº1, Parcela N³943, P.O. Box: 264, Maputo, Mozambique. ${ }^{2}$ Faculty of Medicine, Eduardo Mondlane University (UEM), Maputo, Mozambique. ${ }^{3}$ Unidade de Microbiologia Médica, Instituto de Higiene e Medicina Tropical (IHMT)/Universidade Nova de Lisboa (NOVA), and Global Health and Tropical Medicine (GHTM) Research Centre, Lisbon, Portugal. ${ }^{4}$ Unidade de Parasitologia Médica, Instituto de Higiene e Medicina Tropical (IHMT)/Universidade Nova de Lisboa (NOVA), and Global Health and Tropical Medicine (GHTM) Research Centre, Lisbon, Portugal. ${ }^{5}$ Faculty of Veterinary Sciences Pretoria, University of Pretoria (UP), Pretoria, South Africa.

${ }^{6}$ Faculty of Veterinary, Eduardo Mondlane University (UEM), Maputo, Mozambique.

Received: 8 April 2020 Accepted: 14 July 2020 Published online: 28 July 2020

\section{References}

1. Campbell-Lendrum D, Manga L, Bagayoko M, Sommerfeld J. Climate change and vector-borne diseases: what are the implications for public health research and policy? Philos Trans R Soc Lond B Biol Sci. 2015;370.

2. Bhatt $S$, Gething PW, Brady OJ, Messina JP, Farlow AW, Moyes $C L$, et al. The global distribution and burden of denque. Nature. 2013:496(7446):504-7.

3. WHO. Weekly bulletin on outbreaks and other emergencies vol. week 31: 29 July - 4 august 2019. Geneva: WHO; 2019.

4. Zhang $Y Z$, Shi M, Holmes EC. Using metagenomics to characterize an expanding Virosphere. Cell. 2018;172(6):1168-72.

5. Desnues C, Raoult D. Inside the lifestyle of the virophage. Intervirology. 2010;53(5):293-303.

6. Abrahao J, Silva L, Silva LS, Khalil JYB, Rodrigues R, Arantes T, et al. Tailed giant Tupanvirus possesses the most complete translational apparatus of the known virosphere. Nat Commun. 2018;9(1):749.

7. Kauffman KM, Hussain FA, Yang J, Arevalo P, Brown JM, Chang WK, et al. A major lineage of non-tailed dsDNA viruses as unrecognized killers of marine bacteria. Nature. 2018;554(7690):118-22.

8. Li CX, Shi M, Tian JH, Lin XD, Kang YJ, Chen LJ, et al. Unprecedented genomic diversity of RNA viruses in arthropods reveals the ancestry of negative-sense RNA viruses. eLife. 2015;4.

9. Shi M, Lin XD, Tian JH, Chen LJ, Chen X, Li CX, et al. Redefining the invertebrate RNA virosphere. Nature. 2016;540(7634):539-43.

10. Zhang $Y Z$, Wu WC, Shi M, Holmes EC. The diversity, evolution and origins of vertebrate RNA viruses. Curr Opin Virol. 2018;31:9-16.

11. Gould E, Pettersson J, Higgs S, Charrel R, de Lamballerie X. Emerging arboviruses: why today? One health. 2017:4:1-13. 
12. Bolling BG, Weaver SC, Tesh RB, Vasilakis N. Insect-specific virus discovery: significance for the arbovirus community. Viruses. 2015;7(9):4911-28.

13. Calisher $\mathrm{CH}$, Higgs S. The discovery of arthropod-specific viruses in hematophagous arthropods: an open door to understanding the mechanisms of arbovirus and arthropod evolution? Annu Rev Entomol. 2018;63:87-103.

14. Junglen S, Drosten C. Virus discovery and recent insights into virus diversity in arthropods. Curr Opin Microbiol. 2013;16(4):507-13.

15. Marklewitz M, Junglen S. Evolutionary and ecological insights into the emergence of arthropod-borne viruses. Acta Trop. 2019;190:52-8.

16. Higa Y, Abilio AP, Futami K, Lazaro MA, Minakawa N, Gudo ES. Abundant Aedes (Stegomyia) aegypti aegypti mosquitoes in the 2014 dengue outbreak area of Mozambique. Trop Med Health. 2015;43(2):107-9.

17. Massangaie M, Pinto G, Padama F, Chambe G, da Silva M, Mate I, et al. Clinical and epidemiological characterization of the first recognized outbreak of dengue virus-type 2 in Mozambique, 2014. Am J Trop Med Hyg. 2016;94(2):413-6.

18. Fafetine J, Neves L, Thompson PN, Paweska JT, Rutten VP, Coetzer JA Serological evidence of Rift Valley fever virus circulation in sheep and goats in Zambezia Province, Mozambique. PLoS Negl Trop Dis. 2013;7(2):e2065.

19. Fafetine JM, Tijhaar E, Paweska JT, Neves LC, Hendriks J, Swanepoel R, et al. Cloning and expression of Rift Valley fever virus nucleocapsid (N) protein and evaluation of a N-protein based indirect ELISA for the detection of specific IgG and lgM antibodies in domestic ruminants. Vet Microbiol. 2007; 121(1-2):29-38.

20. Gudo ES, Pinto G, Weyer J, le Roux C, Mandlaze A, José AF, et al. Serological evidence of rift valley fever virus among acute febrile patients in southern Mozambique during and after the 2013 heavy rainfall and flooding: implication for the management of febrile illness. Virol J. 2016;13(96).

21. Gubler DJ, Sather GE, Kuno G, Cabral JR. Dengue 3 virus transmission in Africa. Am J Trop Med Hyg. 1986;35(6):1280-4.

22. Gudo ES, Pinto G, Vene S, Mandlaze A, Muianga AF, Cliff J, et al. Serological evidence of chikungunya virus among acute febrile patients in southern Mozambique. PLoS Negl Trop Dis. 2015;9(10).

23. Mugabe VA, Ali S, Chelene I, Monteiro VO, Guiliche O, Muianga AF, et al. Evidence for chikungunya and dengue transmission in Quelimane, Mozambique: results from an investigation of a potential outbreak of chikungunya virus. PLoS One. 2018;13(2):e0192110.

24. Bogoch II, Brady OJ, Kraemer MU, German M, Creatore MI, Brent S, et al. Potential for Zika virus introduction and transmission in resource-limited countries in Africa and the Asia-Pacific region: a modelling study. Lancet Infect Dis. 2016;16:1237-45.

25. Samy AM, Thomas SM, Wahed AA, Cohoon KP, Peterson AT. Mapping the global geographic potential of Zika virus spread. Mem Inst Oswaldo Cruz. 2016;111(9):559-60.

26. Gudo ES, Falk Kl, Ali S, Muianga AF, Monteiro V, Cliff J. A historic report of Zika in Mozambique: implications for assessing current risk. PLoS Negl Trop Dis. 2016;10.

27. Cugola FR, Fernandes IR, Russo FB, Freitas BC, Dias JL, Guimaraes KP, et al. The Brazilian Zika virus strain causes birth defects in experimental models. Nature. 2016;534(7606):267-71.

28. Worth CB, De Meillon B. Culicine mosquitoes (Diptera:Culicidae) recorded from the province of Mozambique (Portuguese East Africa) and their relation to arthropod-borne virus. Anais Inst Med Trop. 1960;17:231-56.

29. Cholleti H, Hayer J, Abilio AP, Mulandane FC, Verner-Carlsson J, Falk Kl, et al. Discovery of novel viruses in mosquitoes from the Zambezi Valley of Mozambique. PLoS One. 2016;11(9):e0162751.

30. Kampango A, Abilio AP. The Asian tiger hunts in Maputo city--the first confirmed report of Aedes (Stegomyia) albopictus (Skuse, 1895) in Mozambique. Parasit Vectors. 2016;9:76.

31. Abilio AP, Abudasse G, Kampango A, Candrinho B, Sitoi S, Luciano J, et al. Distribution and breeding sites of Aedes aegypti and Aedes albopictus in 32 urban/peri-urban districts of Mozambique: implication for assessing the risk of arbovirus outbreaks. PLoS Negl Trop Dis. 2018;12(9):e0006692.

32. Kuno G, Chang GJ, Tsuchiya KR, Karabatsos N, Cropp CB. Phylogeny of the genus Flavivirus. J Virol. 1998;72(1):73-83.

33. Crochu S, Cook S, Attoui H, Charrel RN, De Chesse R, Belhouchet M, et al. Sequences of flavivirus-related RNA viruses persist in DNA form integrated in the genome of Aedes spp. mosquitoes. J Gen Virol. 2004;85(Pt 7):1971-80.

34. Roiz D, Vazquez A, Rosso F, Arnoldi D, Girardi M, Cuevas L, et al. Detection of a new insect flavivirus and isolation of Aedes flavivirus in northern Italy. Parasit Vectors. 2012;5:223.
35. Roiz D, Vazquez A, Seco MP, Tenorio A, Rizzoli A. Detection of novel insect flavivirus sequences integrated in Aedes albopictus (Diptera: Culicidae) in northern Italy. Virol J. 2009;6:93.

36. Sanchez-Seco MP, Rosario D, Quiroz E, Guzman G, Tenorio A. A generic nested-RT-PCR followed by sequencing for detection and identification of members of the alphavirus genus. J Virol Methods. 2001;95(1-2):153-61.

37. Sall AA, Macondo EA, Sène OK, Diagne M, Sylla R, Mondo M, et al. Use of reverse transcriptase $P C R$ in early diagnosis of Rift Valley fever. Clin Diagn Lab Immunol. 2002;9(3):713-5.

38. Matsuno K, Weisend C, Kajihara M, Matysiak C, Williamson BN, Simuunza M, et al. Comprehensive molecular detection of tick-borne phleboviruses leads to the retrospective identification of taxonomically unassigned bunyaviruses and the discovery of a novel member of the genus phlebovirus. J Virol. 2015;89(1):594-604.

39. Silva M, Morais P, Maia C, de Sousa CB, de Almeida APG, Parreira R. A diverse assemblage of RNA and DNA viruses found in mosquitoes collected in southern Portugal. Virus Res. 2019;274:197769.

40. Sadeghi $M$, Altan E, Deng X, Barker CM, Fang Y, Coffey LL, et al. Virome of $>12$ thousand Culex mosquitoes from throughout California. Virology. 2018; 523:74-88.

41. Chandler JA, Liu RM, Bennett SN. RNA shotgun metagenomic sequencing of northern California (USA) mosquitoes uncovers viruses, bacteria, and fungi. Front Microbiol. 2015;6:185.

42. Gudo ES, Lesko B, Vene S, Lagerqvist N, Candido SI, Razao de Deus N, et al. Seroepidemiologic screening for zoonotic viral infections, Maputo, Mozambique. Emerg Infect Dis. 2016;22(5):915-7.

43. Fafetine JM, Coetzee P, Mubemba B, Nhambirre O, Neves L, Coetzer JA, et al. Rift Valley fever outbreak in livestock, Mozambique, 2014. Emerg Infect Dis. 2016;22(12):2165-7.

44. Hermanns K, Zirkel F, Kopp A, Marklewitz M, Rwego IB, Estrada A, et al. Discovery of a novel alphavirus related to Eilat virus. J Gen Virol. 2017;98(1):43-9.

45. Nasar F, Palacios G, Gorchakov RV, Guzman H, Da Rosa AP, Savji N, et al. Eilat virus, a unique alphavirus with host range restricted to insects by RNA replication. Proc Natl Acad Sci U S A. 2012;109(36):14622-7.

46. Vazquez A, Sanchez-Seco MP, Palacios G, Molero F, Reyes N, Ruiz S, et al. Novel flaviviruses detected in different species of mosquitoes in Spain. Vector Borne Zoonotic Dis. 2012;12(3):223-9.

47. Cook S, Holmes EC. A multigene analysis of phylogenetic relationships among the flaviviruses (family: Flaviviridae) and the evolution of vector transmission, vol. 151; 2006.

48. Cook S, Moureau G, Kitchen A, Gould EA, de Lamballerie X, Holmes EC, et al Molecular evolution of the insect-specific flaviviruses. J Gen Virol. 2012;93(Pt 2):223-34

49. Hegde S, Rasgon JL, Hughes GL. The microbiome modulates arbovirus transmission in mosquitoes. Curr Opin Virol. 2015;15:97-102.

50. Goic B, Vodovar N, Mondotte JA, Monot C, Frangeul L, Blanc $H$, et al. RNA-mediated interference and reverse transcription control the persistence of RNA viruses in the insect model drosophila. Nat Immunol. 2013:14(4):396-403.

51. White SK, Lednicky JA, Okech BA, Morris JG Jr, Dunford JC. Spondweni virus in field-caught Culex quinquefasciatus mosquitoes, Haiti, 2016. Emerg Infect Dis. 2018;24(9):1765-7

52. Beranek MD, Gallardo R, Almiron WR, Contigiani MS. First detection of Mansonia titillans (Diptera: Culicidae) infected with St. Louis encephalitis virus (Flaviviridae: Flavivirus) and Bunyamwera serogroup (Peribunyaviridae: Orthobunyavirus) in Argentina. J Vector Ecol. 2018; 43(2):340-3.

53. Colmant AMG, Hobson-Peters J, Bielefeldt-Ohmann H, van den Hurk AF, Hall-Mendelin S, Chow WK, et al. A new clade of insect-specific Flaviviruses from Australian anopheles mosquitoes displays species-specific host restriction. mSphere. 2017;2(4).

54. Lelieveld SH, Veltman JA, Gilissen C. Novel bioinformatic developments for exome sequencing. Hum Genet. 2016;135(6):603-14

55. Prow NA, Mah MG, Deerain JM, Warrilow D, Colmant AMG, O'Brien CA, et al. New genotypes of Liao ning virus (LNV) in Australia exhibit an insectspecific phenotype. J Gen Virol. 2018;99(4):596-609.

56. Vasilakis N, Guzman H, Firth C, Forrester NL, Widen SG, Wood TG, et al. Mesoniviruses are mosquito-specific viruses with extensive geographic distribution and host range. Virol J. 2014;11:97.

57. Roundy CM, Azar SR, Rossi SL, Weaver SC, Vasilakis N. Insect-specific viruses: a historical overview and recent developments. Adv Virus Res. 2017;98:119-46. 
58. Calzolari M, Ze-Ze L, Vazquez A, Sanchez Seco MP, Amaro F, Dottori M. Insect-specific flaviviruses, a worldwide widespread group of viruses only detected in insects. Infect Genet Evol. 2016;40:381-8.

59. Gillies MT, Coetzee M. A supplement to the Anophelinae of Africa south of the Sahara, vol. 55. Johannesburg; Sounth African Institute For Medical Research; 1987.

60. Jupp PG. Mosquitoes of southern Africa. Pretoria: Ekogilde Publishers; 1986.

61. Sall AA, Thonnon J, Sene OK, Fall A, Ndiaye M, Baudez B, et al. Single-tube and nested reverse transcriptase-polymerase chain reaction for detection of Rift Valley fever virus in human and animal sera. J Virol Methods. 2001;91(1): 85-92.

62. Pereira A, Figueira L, Nunes M, Esteves A, Cotao AJ, Vieira ML, et al. Multiple Phlebovirus (Bunyaviridae) genetic groups detected in Rhipicephalus, Hyalomma and Dermacentor ticks from southern Portugal. Ticks Tick-Borne Dis. 2017:8(1):45-52.

63. Carapeta S, Do Bem B, McGuinnes J, Esteves A, Abecasis A, Almeida APG, et al. Negeviruses found in multiple species of mosquitoes from southern Portugal: isolation, genetic diversity, and replication in insect cell culture. Virology. 2015;483:318-28.

64. Katoh K, Standley DM. MAFFT multiple sequence alignment software version 7: improvements in performance and usability. Mol Biol Evol. 2013; 30(4):772-80.

65. Castresana J. Selection of conserved blocks from multiple alignments for their use in phylogenetic analysis. Mol Biol Evol. 2000;17(4):540-52.

66. Hall TA. BioEdit: a user-friendlybiological sequence alignment editor and analysis program for windows 95/98/NT. Nucleic Acids Symp. 1999:41:4.

67. Tamura K, Stecher G, Peterson D, Filipski A, Kumar S. MEGA6: molecular evolutionary genetics analysis version 6.0. Mol Biol Evol. 2013;30:2725-9.

68. Drummond AJ, Suchard MA, Xie D, Rambaut A. Bayesian phylogenetics with BEAUti and the BEAST 1.7. Mol Biol Evol. 2012;29(8):1969-73.

69. Ho SY, Phillips MJ, Drummond AJ, Cooper A. Accuracy of rate estimation using relaxed-clock models with a critical focus on the early metazoan radiation. Mol Biol Evol. 2005:22(5):1355-63.

\section{Publisher's Note}

Springer Nature remains neutral with regard to jurisdictional claims in published maps and institutional affiliations.

Ready to submit your research? Choose BMC and benefit from:

- fast, convenient online submission

- thorough peer review by experienced researchers in your field

- rapid publication on acceptance

- support for research data, including large and complex data types

- gold Open Access which fosters wider collaboration and increased citations

- maximum visibility for your research: over $100 \mathrm{M}$ website views per year

At $\mathrm{BMC}$, research is always in progress.

Learn more biomedcentral.com/submissions 